\title{
Keplerian squeezed states and Rydberg wave packets
}

\author{
Robert Bluhm, ${ }^{1}$ V. Alan Kostelecký, ${ }^{2}$ and Bogdan Tudose ${ }^{2}$ \\ ${ }^{1}$ Physics Department, Colby College, Waterville, Maine 04901 \\ ${ }^{2}$ Physics Department, Indiana University, Bloomington, Indiana 47405
}

(Received 28 August 1995)

\begin{abstract}
We construct minimum-uncertainty solutions of the three-dimensional Schrödinger equation with a Coulomb potential. These wave packets are localized in radial and angular coordinates and are squeezed states in three dimensions. They move on elliptical Keplerian trajectories and are appropriate for the description of the corresponding Rydberg wave packets, the production of which is the focus of current experimental effort. We extend our analysis to incorporate the effects of quantum defects in alkali-metal atoms, which are used in experiments.
\end{abstract}

PACS number(s): 32.80.Bx, 03.65.-w

\section{INTRODUCTION}

At an early stage in the development of quantum mechanics, Schrödinger attempted to find nonspreading wave-packet solutions for a quantum-mechanical particle evolving along a classical trajectory. He succeeded for the case of a particle subject to a harmonic-oscillator potential, obtaining a solution that follows the classical oscillatory motion without changing shape [1]. This solution is now called a coherent state [2]. Schrödinger also attempted to find analogous solutions for the Coulomb potential, without success [3]. Many authors since have discussed this issue, and it is now known that there are no exact coherent states for the Coulomb problem [4-9].

Although the original Schrödinger problem for the Coulomb potential has no solution, one can nonetheless obtain minimum-uncertainty wave packets exhibiting many features of the corresponding classical motion. For example, it has recently been shown that a type of squeezed state called an elliptical squeezed state (ESS) is a minimum-uncertainty wave packet for the planar Coulomb problem [10]. An ESS is localized in both the radial and angular coordinates, and it travels along a Keplerian ellipse in two dimensions. The radial part of the initial solution is a radial squeezed state (RSS), which minimizes the uncertainty relation for a set of radial variables that express the Coulomb problem in a form similar to that of an oscillator $[11,12]$. The angular part of the initial solution is a circular squeezed state (CSS), which minimizes the uncertainty relation between the angular momentum $L$ and a suitable angular-coordinate operator $[10,13]$.

The primary goal of this paper is to extend the ESS construction to three dimensions and thereby to provide a class of wave packets coming as close as possible to a solution of the original Schrödinger problem for the Coulomb potential. We call these wave packets Keplerian squeezed states (KSS). We show that the KSS are minimum-uncertainty wave packets that are localized in all three dimensions and that travel along a Keplerian ellipse. Three-dimensional wave packets of this kind are of particular interest at present because experiments using short-pulsed lasers are attempting to produce Rydberg wave packets that move along elliptical orbits.

To date, experiments that have detected the classical os- cillation of a wave packet in a Coulomb potential have been performed using purely radial wave packets consisting of a superposition of $n$ states with $l$ fixed, typically, to a $p$ state [14-19]. These states follow the initial classical motion but also exhibit distinctive quantum-mechanical features. For example, the uncertainty product oscillates periodically as a function of time. This is a characteristic of squeezed states [20], and indeed the RSS provide a good analytical description of wave packets of this type. The wave packets also exhibit quantum interference effects, as they undergo a series of collapses and full and fractional revivals and superrevivals [21-26].

To generate a three-dimensional wave packet localized in radial and both angular coordinates, a superposition of $n, l$, and $m$ levels must be created. This requires the presence of additional fields during the excitation process. One proposal for achieving this involves using a short electric pulse to convert an angular state into a localized Rydberg wave packet moving on a circular orbit [27]. An additional weak electric field could then distort the orbit into an ellipse of arbitrary eccentricity. We expect the motion of these wave packets to be well described by the KSS we present here.

The construction of the KSS requires the identification of angular operators appropriate to a problem with spherical symmetry. These can then be used to obtain a class of squeezed states, called spherical squeezed states (SSS), that minimize uncertainty products for the angular variables. This construction and the basic properties of the SSS are presented in Sec. II.

Section III gives the construction of the KSS. At the initial time, the KSS may be written as products of RSS with SSS. The resulting wave packet is localized in all three dimensions and consists of a superposition over the three quantum numbers $n, l$, and $m$. In this section, we also calculate expectation values for physical quantities characterizing the initial wave packet, and we discuss its time evolution.

Section IV outlines how the KSS can be generalized to include the effects of quantum defects. This is necessary because experiments performed on Rydberg wave packets generally use alkali-metal atoms rather than hydrogen.

Section V contains a summary of our results. Some mathematical formulas used in deriving expectation values in Sec. II are given in the Appendix. Throughout this paper, we use 
atomic units with $\hbar=m=e=1$.

\section{SPHERICAL SQUEEZED STATES}

In this section, we obtain the spherical squeezed states. These are squeezed states for angular coordinates on a unit sphere. Section II A discusses the choice of angular variables used in the construction. Section II B gives the derivation of the SSS, and Sec. III C examines their properties.

\section{A. Quantum angular variables on the sphere}

Classically, the standard angular coordinates on the unit sphere are the spherical polar coordinates $\theta$ and $\phi$, which can be taken as defined in the intervals $0 \leqslant \theta \leqslant \pi$ and $-\pi \leqslant \phi<\pi$, respectively. At the quantum level, however, $\theta$ are $\phi$ are not well suited as coordinate operators. Some of the difficulties stem from problems already appearing in the planar case. For example, if $\phi$ is taken as periodic, the discontinuity at $\phi=\pi$ causes complications when $\phi$ is acted upon by derivative operators such as the $z$ component $L_{3}$ of the angular momentum $\vec{L}$. The alternative assumption of a continuous angle $\phi$ in the interval $-\infty<\phi<\infty$ also introduces complications because this variable is not periodic and hence is not observable in the Hilbert space for which $L_{3}=-i \partial_{\phi}$ is Hermitian. In the present instance, the use of $\phi$ in conjunction with $\theta$ on the unit sphere raises further complexities because $\phi$ is not well defined at the poles $\theta=0, \pi$.

Difficulties with angular coordinates on the circle have been widely discussed in the literature [28]. In addressing the Schrödinger problem for the planar Coulomb potential [10] and in a related work on minimum-uncertainty angular packets [13], we followed Ref. [29] in circumventing these and other difficulties by replacing $\phi$ with two quantum coordinate operators, $\sin \phi$ and $\cos \phi$, which are both continuous and periodic. Use of these variables permitted a completely satisfactory resolution to the essential issues.

In the present work, we follow a similar strategy. We introduce three angular-coordinate operators $a_{j}, j=1,2,3$, via their matrix elements obtained by insertion of the three continuous and periodic coordinate functions $f_{j}=(\sin \theta \cos \phi, \sin \theta \sin \phi, \cos \theta), \quad$ respectively, in the Hilbert-space inner product

$$
\left\langle\psi_{1}\left|a_{j}\right| \psi_{2}\right\rangle=\int_{-\pi}^{\pi} \int_{0}^{\pi} d \Omega \psi_{1}^{*} f_{j} \psi_{2},
$$

where $d \Omega$ is an element of solid angle on the sphere. They obey the relation $\Sigma_{j} a_{j}^{2}=1$. Classically, they are a natural choice corresponding to the identification $(x, y, z) \rightarrow(\sin \theta \cos \phi, \sin \theta \sin \phi, \cos \theta)$, which uniquely covers the unit sphere. Moreover, this choice reduces in the limit $\theta \rightarrow \pi / 2$ to the planar case.

The quantum operators $a_{j}$ and the components $L_{k}$ of the angular-momentum operator $\vec{L}$ obey the commutation relations

$$
\left[a_{j}, L_{k}\right]=i \epsilon_{j k l} a_{l}
$$

Each of these commutators has an associated uncertainty relation that must be considered in the search for minimumuncertainty solutions.

The number of nontrivial relations can be substantially reduced by stipulating certain initial conditions on the desired wave packet. Without loss of generality, we take the initial minimum-uncertainty configuration to be a wave packet localized about the positive $x$ axis. Since we seek a packet moving along an orbit in the $x-y$ plane, we also assume the initial shape is reflection-symmetric about this plane. It is also physically reasonable to suppose that the initial packet is reflection-symmetric about the $x-z$ plane. These requirements impose the coordinate conditions

$$
\left\langle a_{2}\right\rangle=\left\langle a_{3}\right\rangle=0, \quad\left\langle a_{1}\right\rangle>0
$$

and the angular-momentum conditions

$$
\left\langle L_{1}\right\rangle=\left\langle L_{2}\right\rangle=0 .
$$

The conditions (3) leave only two nontrivial uncertainty relations to be considered. They are

$$
\Delta a_{2} \Delta L_{3} \geqslant \frac{1}{2}\left|\left\langle a_{1}\right\rangle\right|
$$

and

$$
\Delta a_{3} \Delta L_{2} \geqslant \frac{1}{2}\left|\left\langle a_{1}\right\rangle\right| \text {. }
$$

\section{B. Construction of SSS}

The CSS are defined as states minimizing the relation (5) in the $x-y$ plane [10]. Requiring that the SSS reduce to the CSS in the planar limit $\theta \rightarrow \pi / 2$ means that (5) must be minimized in three dimensions also. Our construction of the SSS in this section therefore begins with this minimization, after which we return to the remaining relation (6).

A wave function $\chi(\theta, \phi)$ minimizing Eq. (5) obeys the differential equation

$$
a_{2} \chi(\theta, \phi)=-i \frac{1}{\delta}\left(L_{3}-\left\langle L_{3}\right\rangle\right) \chi(\theta, \phi),
$$

where the squeezing $\delta$ in the angular coordinates is a real constant given by

$$
\frac{1}{\delta} \equiv \frac{\Delta a_{2}}{\Delta L_{3}}=\frac{2\left(\Delta a_{2}\right)^{2}}{\left|\left\langle a_{1}\right\rangle\right|} \geqslant 0 .
$$

As expected, setting $\theta \rightarrow \pi / 2$ reduces Eqs. (7) and (8) to the defining equations for the CSS. Fixing $\left\langle L_{3}\right\rangle=\beta$, a real parameter, Eq. (7) becomes

$$
\left(\partial_{\phi}+\delta \sin \theta \sin \phi-i \beta\right) \chi(\theta, \phi)=0 .
$$

The solution to this equation is

$$
\chi(\theta, \phi)=N \exp [\delta \sin \theta \cos \phi+i \beta \phi+f(\theta)],
$$

where $f(\theta)$ is an undetermined complex function of $\sin \theta$ and $\cos \theta$. For $\chi(\theta, \phi)$ to be single valued, $\beta$ must be an integer. This follows from the general result [13] that minimum- 
uncertainty angular wave packets must have integer expectation values $\left\langle L_{3}\right\rangle$ of the angular momentum.

We next address the issue of the role of the second uncertainty relation (6). An immediate question is whether the function $f(\theta)$ may be chosen so that $\chi(\theta, \phi)$ also minimizes (6). This would require that, in addition to (9), $\chi(\theta, \phi)$ must satisfy the differential equation

$$
\cos \theta \chi(\theta, \phi)=i \frac{1}{\delta^{\prime}}\left(-i \cos \phi \partial_{\theta}+i \cot \theta \sin \phi \partial_{\phi}\right) \chi(\theta, \phi),
$$

where $\delta^{\prime}$ is a second squeezing parameter defined by $1 / \delta^{\prime}=\Delta a_{3} / \Delta L_{2}$. However, substituting (10) into (11) produces an equality only if $\delta=\delta^{\prime}, \beta=0$, and $f(\theta)$ is a constant. Physically, this means the squeezing ratios are equal, $\Delta a_{2} / \Delta L_{3}=\Delta a_{3} / \Delta L_{2}$, and that the mean angular momentum vanishes, $\left\langle L_{1}\right\rangle=\left\langle L_{2}\right\rangle=\left\langle L_{3}\right\rangle=0$. However, this is unacceptable because we seek solutions with a nonzero value for $\left\langle L_{3}\right\rangle$. Indeed, the two uncertainty relations (5) and (6) can be satisfied simultaneously only for states symmetric under rotations about the $x$ axis. The rotational symmetry must therefore be broken, and the relation (6) cannot be minimized.

One simple way to break the rotational symmetry would be to keep $\left\langle L_{3}\right\rangle \neq 0$ in Eq. (10) but to set $f(\theta) \equiv 0$. The function $\chi(\theta, \phi)$ then would take a simplified form that reduces directly to a CSS as $\theta \rightarrow \pi / 2$. This choice has the added elegant feature that expectation values would take the same form as those for the CSS given in Ref. [10], except with modified Bessel functions $I_{\nu}$ replaced by modified spherical Bessel functions $i_{\nu}$ with different arguments. However, wave packets of this type are also unacceptable because certain physical expectation values diverge. For example, $\left\langle\vec{L}^{2}-L_{3}^{2}\right\rangle$ diverges.

Obtaining an acceptable minimum-uncertainty wave packet evidently requires setting $\beta \neq 0$ and deriving a suitable nonconstant $f(\theta)$. Further physical input is needed to accomplish this. Note that, whatever its form, the function $f(\theta)$ must satisfy the limit $f(\theta) \rightarrow 0$ as $\theta \rightarrow \pi / 2$, so that $\chi(\theta, \phi)$ reduces correctly to a CSS. Furthermore, the reflection symmetry about the $x-y$ plane suggests that $f=f(\sin \theta)$, independent of $\cos \theta$.

The function $f(\theta)$ controls the spread transverse to the orbit of the initial wave packet. This spread should depend on the value of $\beta=\left\langle L_{3}\right\rangle$, since physically as $\left\langle L_{3}\right\rangle$ increases the wave packet is expected to become more confined to a region around the $x-y$ plane. For simplicity, we take the spread as independent of the spread around the orbit, which is controlled by the parameter $\delta$. It turns out that this assumption suffices for our purposes. If it is relaxed, the resulting wave packets have a significantly more complicated analytical structure. In what follows, we therefore permit $f(\theta)$ to depend on $\beta$, but we require it to be independent of $\delta$.

A wave packet following a Keplerian orbit with maximal confinement to the $x-y$ plane should have minimal expectation value $\left|\left\langle\vec{L}^{2}-L_{3}^{2}\right\rangle\right|$. We can use this physical condition to obtain $f(\theta)$, as follows. Since $f(\theta)$ is independent of $\delta$, the limit $\delta \rightarrow 0$ can be imposed in Eq. (10) without loss of generality. In this limit, the uncertainty $\Delta L_{3}$ vanishes because $\chi(\theta, \phi)$ becomes an eigenstate of $L_{3}$ with eigenvalue $m=\beta$. Expanding $\chi(\theta, \phi)$ in terms of spherical harmonics $Y_{l m}(\theta, \phi)$ with $m=\beta$ gives

$$
\left.\chi(\theta, \phi)\right|_{\delta \rightarrow 0}=\sum_{l \geqslant \beta} c_{l} Y_{l \beta}(\theta, \phi) .
$$

Then, calculating the expectation value $\left|\left\langle\vec{L}^{2}-L_{3}^{2}\right\rangle\right|$ in the state $\left.\chi(\theta, \phi)\right|_{\delta \rightarrow 0}$ shows that it is minimized when the coefficients $c_{l}$ in Eq. (12) are proportional to $\delta_{l \beta}$. The sum reduces to a single term proportional to $Y_{\beta \beta}$ and hence proportional to $\sin ^{\beta} \theta \exp (i \beta \phi)$. This implies that $\exp [f(\theta)]$ $=\sin ^{\beta} \theta$. As expected, $f(\theta)$ is a function of $\sin \theta$ and satisfies the condition $f(\pi / 2)=0$. Moreover, this particular functional form leads to an SSS without singularities at the poles and hence with finite physical expectation values.

Combining this result with Eq. (10) produces a set of minimum-uncertainty states satisfying the requirements for SSS. We therefore take the SSS to be given by

$$
\chi(\theta, \phi)=N \sin ^{\beta} \theta \exp (\delta \sin \theta \cos \phi+i \beta \phi) .
$$

\section{Features of SSS}

We next calculate the normalization constant and some expectation values for the SSS. These are most conveniently specified in terms of a function $A_{j}^{\beta}(\delta)$, defined as

$$
A_{j}^{\beta}(\delta)=2 \pi \int_{0}^{\pi} d \theta(\sin \theta)^{2 \beta+j+1} I_{j}(2 \delta \sin \theta),
$$

where $j$ is an integer and $I_{j}(z)$ is a modified Bessel function of the first kind. The Appendix presents analytical expressions for the functions $A_{j}^{\beta}(\delta)$ and discusses some useful properties.

The normalization constant $N$ is given by

$$
N=\frac{1}{\sqrt{A_{0}^{\beta}(\delta)}} .
$$

The nonvanishing expectation values of the angularcoordinate operators are

$$
\begin{gathered}
\left\langle a_{1}\right\rangle=\frac{A_{1}^{\beta}(\delta)}{A_{0}^{\beta}(\delta)}, \\
\left\langle a_{1}^{2}\right\rangle=\frac{1}{2 A_{0}^{\beta}(\delta)}\left[A_{0}^{\beta+1}(\delta)+A_{2}^{\beta}(\delta)\right], \\
\left\langle a_{2}^{2}\right\rangle=\frac{1}{2 \delta} \frac{A_{1}^{\beta}(\delta)}{A_{0}^{\beta}(\delta)}=\frac{1}{2 \delta}\langle\sin \theta \cos \phi\rangle, \\
\left\langle a_{3}^{2}\right\rangle=\frac{1}{A_{0}^{\beta}(\delta)}\left[A_{0}^{\beta}(\delta)-A_{0}^{\beta+1}(\delta)\right],
\end{gathered}
$$

while some useful expectations involving the angular momentum are

$$
\left\langle L_{3}^{2}\right\rangle=\frac{\delta}{2} \frac{A_{1}^{\beta}(\delta)}{A_{0}^{\beta}(\delta)}+\beta^{2}=\frac{\delta}{2}\langle\sin \theta \cos \phi\rangle+\beta^{2},
$$




$$
\begin{aligned}
\left\langle\vec{L}^{2}\right\rangle= & \beta(\beta+1)-\delta^{2}\left[1-\left\langle(\sin \theta \cos \phi)^{2}\right\rangle\right] \\
& +\frac{2 \delta}{A_{0}^{\beta}(\delta)}\left[(\beta+1) A_{1}^{\beta}(\delta)-\beta A_{1}^{\beta-1}(\delta)\right] .
\end{aligned}
$$

Using the identity (A2) for the $A_{j}^{\beta}(\delta)$ in the Appendix, one can show that $\Sigma_{j} a_{j}^{2}=1$ as required. Note that, in the limit $\delta \rightarrow 0,\left\langle L_{3}^{2}\right\rangle=\beta^{2}$ and $\left\langle\vec{L}^{2}\right\rangle=\beta(\beta+1)$, as is appropriate for an eigenstate with $l=m=\beta$.

We next turn to a discussion of some properties of the SSS. First, expanding $\chi(\theta, \phi)$ in spherical harmonics for the case with $\delta \neq 0$ shows that it is a superposition of both $l$ and $m$ states. The condition $\exp [f(\theta)]=\sin ^{\beta} \theta$ ensures that the squared modulus $\left|c_{l m}\right|^{2}$ of the weighting coefficients $c_{l m}$ in the expansion is maximal for $l=m=\beta$ and falls rapidly away from these values. The rapid decrease indicates that $\left\langle\vec{L}^{2}-L_{3}^{2}\right\rangle$ remains small for $\delta \neq 0$, as expected. The reader interested in the form of the expansion can find it in Eq. (31) of Sec. III.

We have seen that the parameter $\beta$ is the expectation of the $z$ component of the angular momentum. It turns out that the parameter $\delta$ has an interpretation similar to that of its counterpart for the CSS. The uncertainty $\Delta L_{3}$ depends on $\delta$. This may be confirmed in the neighborhood of $x=1$, $y=z=0$ on the unit sphere by expanding the wave packet around $\phi=0$ at $\theta=\pi / 2$. We find $|\chi(\pi / 2, \phi)|^{2} \propto \exp [2 \delta(1-$ $\left.\left.\frac{1}{2} \phi^{2}+\cdots\right)\right]$, which to leading order is a Gaussian in $\phi$ with standard deviation $\sigma=1 / \sqrt{2 \delta}$. Therefore, for larger values of $\delta$ the initial wave packet becomes narrower in $\phi$.

Classically, a particle moving along a trajectory confined to the $x-y$ plane has angular motion depending only on $\phi$. The trajectory is initialized by giving values of $\sin \phi$, the sign of $\cos \phi$, and $L_{3}$. At the quantum level, the initial angular position of an SSS is specified by the expectation values in Eq. (3). As we have seen, the parameter $\beta$ gives the initial angular momentum $\left\langle L_{3}\right\rangle$ and controls the transverse angular spread, while $\delta$ controls the angular spread along the orbit. Compared to the corresponding classical problem, a general quantum packet would need two additional parameters determining the spread on the surface of the sphere. However, our simplifying assumption for $f(\theta)$ means that only one extra parameter is needed to specify the quantum solution for the SSS. A related point is that the SSS and CSS depend on the same number of parameters. This is physically reasonable since both states follow the same Keplerian orbit in the two-dimensional $x-y$ plane.

\section{KEPLERIAN SQUEEZED STATES}

This section discusses the Keplerian squeezed states. Their definition is presented in Sec. III A, where their parameters are determined in terms of specified physical quantities. The time evolution of a KSS is examined in Sec. III B, and an example of a KSS wave packet moving along an elliptical orbit is provided.

\section{A. Construction and specification of KSS}

The SSS solution (13) is a function only of the angular coordinates and has neither time dependence nor dependence on the quantum number $n$. A solution consisting of a product of an SSS with a radial energy eigenstate of given $n$ might be considered, but this produces a stationary state. However, we can create a three-dimensional state moving on a Keplerian orbit by combining an SSS with an RSS.

The RSS are constructed and analyzed in Refs. [11,12]. They are wave packets localized in the radial coordinates that initially undergo oscillatory motion between the inner and outer apsidal points of the corresponding Keplerian ellipse. The period $T_{\mathrm{cl}}$ of the motion is that of a classical particle moving in a Coulomb potential. The construction involves converting the classical effective radial Hamiltonian for the Coulomb potential in terms of conventional radial variables to an oscillator description in terms of new variables. The resulting classical problem is quantized, and wave functions are obtained that minimize the ensuing quantum uncertainty relation. The RSS are given by

$$
\begin{gathered}
\psi(r)=N^{\prime} r^{\alpha} \exp \left[-\gamma_{0} r-i \gamma_{1} r\right], \\
N^{\prime}=\left[\left(2 \gamma_{0}\right)^{2 \alpha+3} / \Gamma(2 \alpha+3)\right]^{1 / 2} .
\end{gathered}
$$

For the initial wave packet, the parameters $\alpha, \gamma_{0}$, and $\gamma_{1}$ determine the radial position, the uncertainty in the radial variables, and the radial momentum. In Refs. [11,12], the RSS are shown to describe a Rydberg wave packet that has been excited by a single short laser pulse. The angular part of the full three-dimensional wave function in this case is fixed to a $p$ state for excitation from the ground state.

A minimum-uncertainty wave packet moving on a Keplerian orbit can be obtained by combining an RSS with an SSS. The resulting wave packet is a KSS. The separability of the full Hamiltonian and the independence of $l$ of the RSS uncertainty in the new radial coordinate together make it possible to minimize simultaneously the uncertainty relations for the new radial variables and those for the angular variables in (5). We therefore can take as an initial wave function the product of $\psi(r)$ in (22) and $\chi(\theta, \phi)$ in (13). The result is a normalized five-parameter family of KSS,

$$
\begin{aligned}
\Psi(r, \theta, \phi)= & \psi(r) \chi(\theta, \phi) \\
= & N N^{\prime} r^{\alpha} \sin ^{\beta} \theta \exp [\delta \sin \theta \cos \phi \\
& \left.-\left(\gamma_{0}+i \gamma_{1}\right) r+i \beta \phi\right]
\end{aligned}
$$

where $N$ is given by Eq. (15) and $N^{\prime}$ is given by Eq. (22). The KSS are minimum-uncertainty wave packets localized in all three dimensions. The choice of the initial angularcoordinate location is implicit in the SSS construction and is specified in Eq. (3).

Expectation values of operators for the KSS can be calculated analytically. Since the radial and angular wave functions separate, the angular operators have the expectation values given in Eqs. (16)-(21). The expectation values for the radial operators are

$$
\langle r\rangle=\frac{2 \alpha+3}{2 \gamma_{0}}, \quad\left\langle\frac{1}{r}\right\rangle=\frac{\gamma_{0}}{\alpha+1},
$$




$$
\begin{gathered}
\left\langle r^{2}\right\rangle=\frac{(\alpha+2)(2 \alpha+3)}{2 \gamma_{0}^{2}}, \quad\left\langle\frac{1}{r^{2}}\right\rangle=\frac{2 \gamma_{0}^{2}}{(\alpha+1)(2 \alpha+1)}, \\
\left\langle p_{r}\right\rangle=-\gamma_{1}, \quad\left\langle p_{r}^{2}\right\rangle=\frac{\gamma_{0}^{2}}{2 \alpha+1}+\gamma_{1}^{2} .
\end{gathered}
$$

The uncertainty for the radial coordinates is

$$
\Delta r \Delta p_{r}=\frac{1}{2}\left(\frac{2 \alpha+3}{2 \alpha+1}\right)^{1 / 2}
$$

The RSS are not minimum-uncertainty states in the variables $r$ and $p_{r}$, which is as expected since the construction minimizes the uncertainty in the new radial variables instead. For large values of $\alpha$, however, $\Delta r \Delta p_{r} \rightarrow \frac{1}{2}$.

The expectation value for the energy $\langle H\rangle$ is obtained using the full Coulomb Hamiltonian and depends on all five of the KSS parameters. It is

$$
\langle H\rangle=\frac{1}{2}\left\langle p_{r}^{2}\right\rangle+\frac{1}{2}\left\langle\frac{1}{r^{2}}\right\rangle\left\langle\vec{L}^{2}\right\rangle-\left\langle\frac{1}{r}\right\rangle .
$$

We next address the issue of initializing a KSS. With the wave packet located at the outer apsidal point of an elliptical orbit, the uncertainty product for a radial Rydberg wave packet is a minimum. Initially imposing the constraints (3) ensures that the ellipse has its semimajor axis aligned along the $x$ axis. The five KSS parameters $\alpha, \beta, \gamma_{0}, \gamma_{1}$, and $\delta$ can then be fixed by specifying the expectations of the radial coordinates $\langle r\rangle$ and $\left\langle p_{r}\right\rangle$, the expectation of the angular momentum $\left\langle L_{3}\right\rangle$, the expectation of the energy $\langle H\rangle$, and the spread $\Delta L_{3}$ in the angular momentum.

The natural choice for the initial expectation value of $p_{r}$ is zero. Similarly, the natural choice for the initial expectation value of $r$ is the outer apsidal point of the orbit, $r_{\text {out }}=n^{2}\left\{1+\sqrt{1-l(l+1) / n^{2}}\right\}$. The expectation $\left\langle L_{3}\right\rangle$ gives the initial angular momentum of the wave packet and its spread transverse to the orbit. The two remaining conditions fix the initial spread of the wave packet in the radial and orbital directions. For the first, a natural choice is to set $\langle H\rangle$ equal to the mean energy of a Rydberg wave packet consisting of a superposition of $n$ states centered on the value $\bar{n}$. A packet of this type is produced by excitation with a short laser pulse tuned to the mean energy $E_{\bar{n}}=-1 / 2 \bar{n}^{2}$. For the second, it suffices to specify the width $\Delta L_{3}$ of the superposition. Note, however, that a single laser pulse cannot excite a wave packet localized in the angular coordinates. Creation of a superposition of angular eigenstates requires additional fields to mix angular-momentum eigenstates.

The full set of conditions sufficient to fix the five KSS parameters are then

$$
\begin{gathered}
\langle r\rangle=r_{\text {out }}, \quad\left\langle p_{r}\right\rangle=0, \quad\left\langle L_{3}\right\rangle=\beta, \\
\langle H\rangle=E_{\bar{n}}, \quad \sqrt{\left\langle L_{3}^{2}\right\rangle-\left\langle L_{3}\right\rangle^{2}}=\Delta L_{3} .
\end{gathered}
$$

These determine the values of $\alpha, \beta, \gamma_{0}, \gamma_{1}$, and $\delta$ in terms of physical parameters in the excitation process.

\section{B. Evolution of KSS}

We next investigate the time evolution of a KSS wave function matched to a Rydberg wave packet at the outer apsidal point of an elliptical orbit, as described above. By construction, the packet is expected to travel along a classical ellipse in the $x-y$ plane.

Since $\left\langle p_{r}\right\rangle=0$ and $\left\langle L_{3}\right\rangle=\beta$, the initial motion of the wave packet for $\beta>0$ is in the direction of increasing $\phi$. The geometry of the ensuing orbit depends on the values of $\bar{n}, \beta$, and $\Delta L_{3}$. The parameter $\beta$ gives the central values of $l$ and $m$. For $\beta \simeq \bar{n}-1$, the KSS orbit becomes circular, with the wave packet propagating at fixed mean radial distance from the origin. As $\beta$ decreases, the orbit becomes elliptical, with the inner apsidal point moving closer to the nucleus. Also, for $\beta \rightarrow 1$ the radial wave function becomes oscillatory as the electron passes close to the nucleus.

To study the time evolution of a KSS, we expand $\Psi(r, \theta, \phi)$ in (23) in terms of energy and angular-momentum eigenstates,

$$
\Psi(r, \theta, \phi, t)=\sum_{n, l, m} c_{n l m} R_{n l}(r) Y_{l m}(\theta, \phi) e^{-i E_{n} t}
$$

The expansion coefficients

$$
c_{n l m}=\left\langle\Psi(r, \theta, \phi, 0) \mid R_{n l}(r) Y_{l m}(\theta, \phi)\right\rangle
$$

can be calculated using Eq. (23) as the initial wave function $\Psi(r, \theta, \phi, 0)$. The radial and angular parts separate, and we may write $c_{n l m}=c_{n l}^{(\mathrm{RSS})} c_{l m}^{(\mathrm{SSS})}$, where $c_{n l}^{(\mathrm{RSS})}=\left\langle\psi(r) \mid R_{n l}(r)\right\rangle$ for $\psi(r)$ in (22) and $c_{l m}^{\text {(SSS) }}=\left\langle\chi(\theta, \phi) \mid Y_{l m}(\theta, \phi)\right\rangle$ for $\chi(\theta, \phi)$ in (13). The coefficients $c_{n l}^{\text {(RSS) }}$ for the radial part of the expansion are given in Eq. (58) of Ref. [12]. The coefficients for the angular expansion can be calculated using (13). The result for $m \geqslant 0$ is

$$
\begin{aligned}
c_{l m}^{(\mathrm{SSS})}=N 4 & \sqrt{\pi} \delta_{0,(l-m) \bmod 2}(-1)^{m}\left(\frac{2 l+1}{4 \pi} \frac{(l-m) !}{(l+m) !}\right)^{1 / 2} \sum_{k=0}^{1 / 2(l-m)} \sum_{p=0}^{\min (m, \beta)} \frac{(-1)^{k}}{2^{k} k !} \\
& \times \frac{(2 l-2 k-1) ! !}{(l-m-2 k) !}(-1)^{p}\left(\begin{array}{c}
\min (m, \beta) \\
p
\end{array}\right) 2^{(l-m+2 p-2 k) / 2} \\
& \times \frac{\Gamma((l-m+2 p-2 k+1) / 2)}{\delta^{(l-m+2 p-2 k) / 2}} i_{(2 \beta+l-3 m+2 p-2 k) / 2}(\delta) .
\end{aligned}
$$




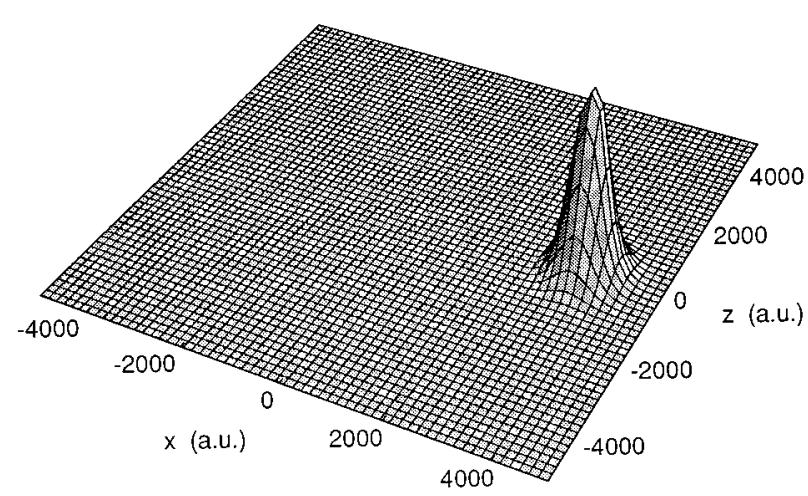

FIG. 1. A slice through the initial KSS wave packet in the $x-z$ plane, which is transverse to the plane of the classical orbit. The quantity $r^{2}|\Psi(r, \theta, \phi)|^{2}$ (in arbitrary units) is plotted as a function of $x$ and $z$ at $t=0$.

Here, $N$ is the SSS normalization constant in (15), min $(m, \beta)$ gives the minimum of the two values $m$ and $\beta$, and $i_{\nu}(z)$ is a modified spherical Bessel function. The appearance of the Kronecker delta $\delta_{0,(l-m) \bmod 2}$ implies that if $l-m$ is an odd integer then $c_{l m}^{(\mathrm{SSS})}=0$. Therefore, the SSS is composed only of eigenstates for which $l-m$ is even. This follows because the associated Legendre functions with odd $l-m$ are odd functions of $\theta$ in the interval $0 \leqslant \theta \leqslant \pi$ centered on $\pi / 2$, while $\chi(\theta, \phi)$ is an even function of $\theta$ in this interval.

As an explicit example of the evolution of a KSS, consider matching $\Psi(r, \theta, \phi, 0)$ to a Rydberg wave packet at the outer apsidal point with $\bar{n}=45,\left\langle L_{3}\right\rangle=30$, and $\Delta L_{3}=2.5$. Using these values and Eqs. (29), we obtain the KSSparameter values $\alpha \simeq 62.846, \beta=30, \gamma_{0} \simeq 0.01834, \gamma_{1}=0$, and $\delta \simeq 12.826$. This gives $\langle r\rangle=r_{\text {out }} \simeq 3508.6$ a.u. and $\left\langle\vec{L}^{2}\right\rangle \simeq 938$.1. Defining a mean value $\bar{l}$ of $l$ from the relation $\left\langle\vec{L}^{2}\right\rangle=\bar{l}(\bar{l}+1)$, we find $\bar{l} \simeq 30.1 \approx \beta$.

The series in (30) may be well approximated by truncating the sum to a finite number of terms with $n$ centered on $\bar{n}$ and $l$ and $m$ centered on $\beta$. In the present example, we allow a spread of ten units in $n$ and $l$ and four units in $m$. This gives 484 coefficients, half of which vanish. We keep the remaining 242 terms in the series and plot twodimensional sections through the KSS as a function of $t$.

Figure 1 presents a slice through the initial KSS in the $x-z$ plane. This slice is transverse to the classical orbit, which lies in the $x-y$ plane. The figure shows that the initial wave packet is localized around a point on the $x$ axis at $r \approx r_{\text {out }}$ and in a narrow range of $\theta$ near $\theta=\pi / 2$, corresponding to $z \approx 0$.

Figure 2(a) presents a slice through the initial wave packet in the plane of the classical orbit. The initial wave packet is located on the positive $x$ axis at the outer apsidal point. Figures 1 and 2(a) taken together show that the initial KSS is localized in all three dimensions.

The classical Keplerian orbit for a particle in a Coulomb potential is $T_{\mathrm{cl}}=2 \pi \bar{n}^{3}$. With $\bar{n}=45$, we obtain $T_{\mathrm{cl}} \simeq 13.4$ psec. Figure 2(b) shows the KSS in the $x-y$ plane at $t=T_{\mathrm{cl}} / 3$. It has moved in the direction of positive $\phi$ and is spreading along the elliptical orbit. In accordance with Kepler's laws, the wave packet moves more slowly near the outer apsidal point than near the inner one. As a result, it has traveled less than $1 / 3$ of the orbital circumference at $t=T_{\mathrm{cl}} / 3$.

Figure 2(c) shows the KSS in the $x-y$ plane at $t=T_{\mathrm{cl}} / 2$. It has spread along the elliptical orbit and is moving more rapidly. The radial distance to the inner apsidal point is $r_{\text {in }} \simeq 536$ a.u. This is sufficiently far from the nucleus for the wave packet to remain localized in $r$, and hence no radial oscillations are apparent.

Figures 2(d) and 2(e) show the slice through the KSS in the $x-y$ plane at the times $t=2 T_{\mathrm{cl}} / 3$ and $t=T_{\mathrm{cl}}$, respectively. The motion slows again as the wave packet approaches the outer apsidal point and becomes more localized. At $t=T_{\mathrm{cl}}$, the wave packet resembles the initial wave packet. However, the motion is not exactly periodic. As time increases, the wave packet collapses and for $t \gg T_{\mathrm{cl}}$ a cycle of revivals and superrevivals commences.

Figure 3 shows the wave packet in the $x-y$ plane at $t=T_{\mathrm{cl}} / 2$, but viewed from a point on the positive $x$ axis looking toward the nucleus. The elliptical shape of the orbit is evident.

\section{INCORPORATION OF QUANTUM DEFECTS}

Experiments studying the behavior of Rydberg wave packets are usually performed using alkali-metal atoms. These have energies given by the Rydberg series $E_{n *}=-1 / 2 n^{* 2}$, where $n^{*}=n-\delta(n, l)$, and $\delta(n, l)$ is a quantum defect. The empirical parameters $\delta(n, l)$ give the energy-level shifts away from hydrogenic values. For large $n$, they approach asymptotic values $\delta(l)$ independent of $n$.

In Ref. [12], it is shown that the RSS construction can be generalized to include the effects of quantum defects. The analysis uses a model called supersymmetry-based quantumdefect theory (SQDT) to describe alkali-metal atoms [30]. This analytical theory for alkali-metal atoms has exact asymptotic quantum-defect energies as eigenvalues. The SQDT eigenfunctions form a complete and orthogonal set.

The SQDT eigenstates are $R_{n * l *}(r) Y_{l m}(\theta, \phi)$, where $n^{*}=n-\delta(l), l^{*}=l-\delta(l)+I(l)$, and $I(l)$ is an integer that depends on $l$. The radial eigenstates $R_{n} * l^{*}(r)$ have the same functional form as the hydrogenic functions $R_{n l}(r)$, but $n$ is replaced by $n^{*}$ and $l$ is replaced by $l^{*}$. The angular wave functions are the usual eigenstates $Y_{l m}(\theta, \phi)$ of the angular momentum.

Our KSS construction in three dimensions can be generalized to include the effects of quantum defects [31]. Since the angular part of the solution in SQDT separates, the SSS wave functions remain unchanged and are given by $\chi(\theta, \phi)$ in Eq. (13). The RSS wave functions $\psi(r)$ are obtained by writing the classical SQDT Hamiltonian in terms of new radial oscillator variables incorporating effects of quantum defects and then finding minimum-uncertainty solutions for the corresponding quantum problem. The resulting wave function $\psi(r)$ has a related functional form to that in Eq. (22) and is discussed in Ref. [12].

A $\operatorname{KSS} \Psi(r, \theta, \phi)$ for alkali-metal atoms is again formed as a product of an $\operatorname{RSS} \psi(r)$ and an $\operatorname{SSS} \chi(\theta, \phi)$. The functional form of the solution is similar to that in Eq. (23) but with suitable replacements for the quantum numbers. To allow for the shifted energy eigenvalues of the alkali-metal atoms, the initialization procedure for the parameters must be 
(a)

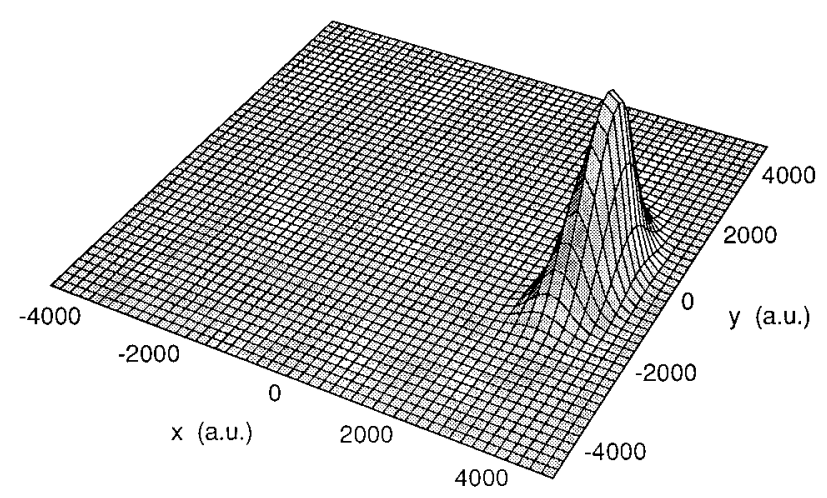

(b)

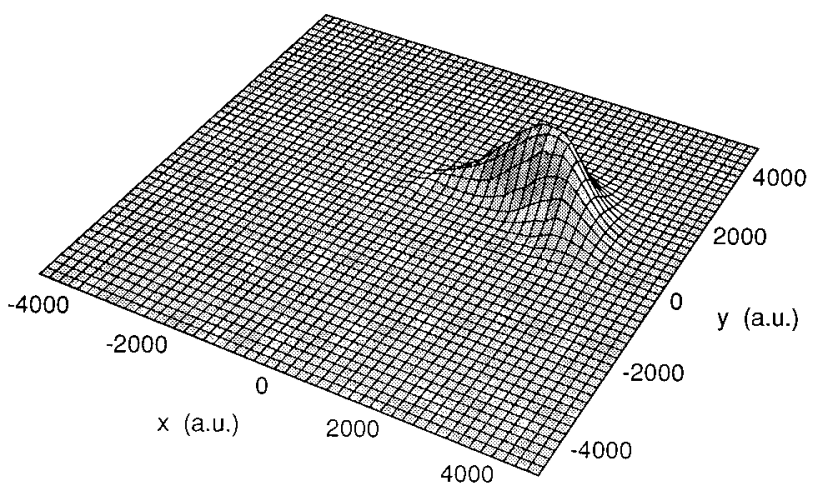

(c)

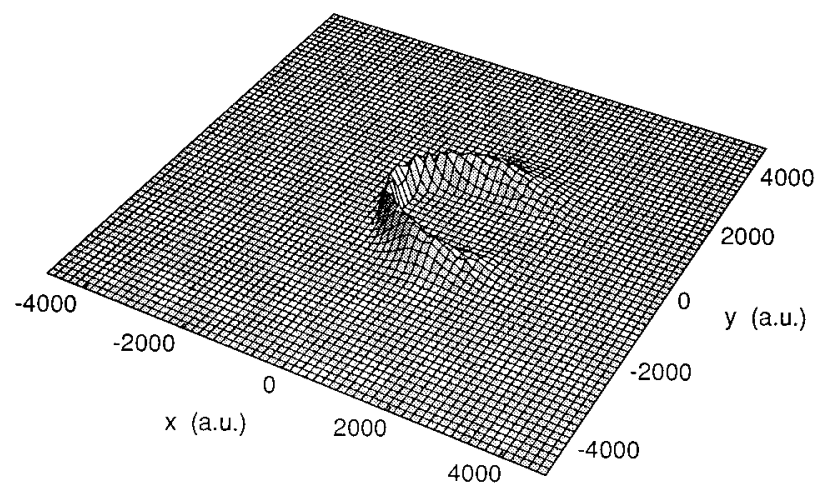

(d)

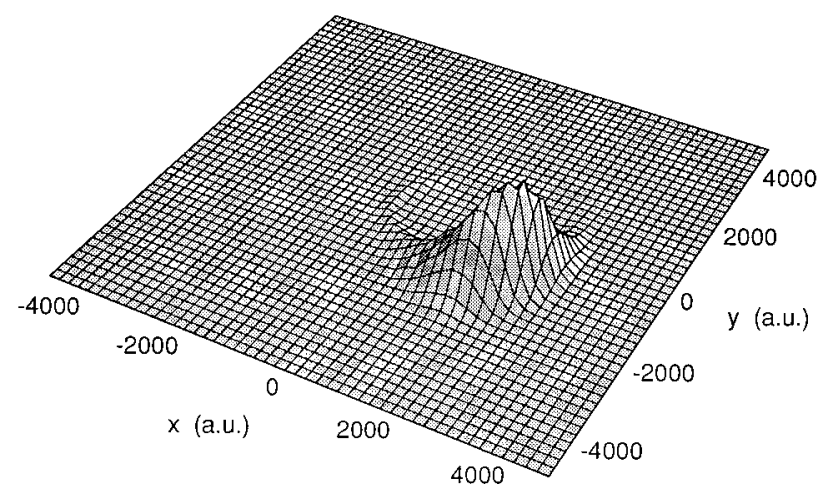

(e)

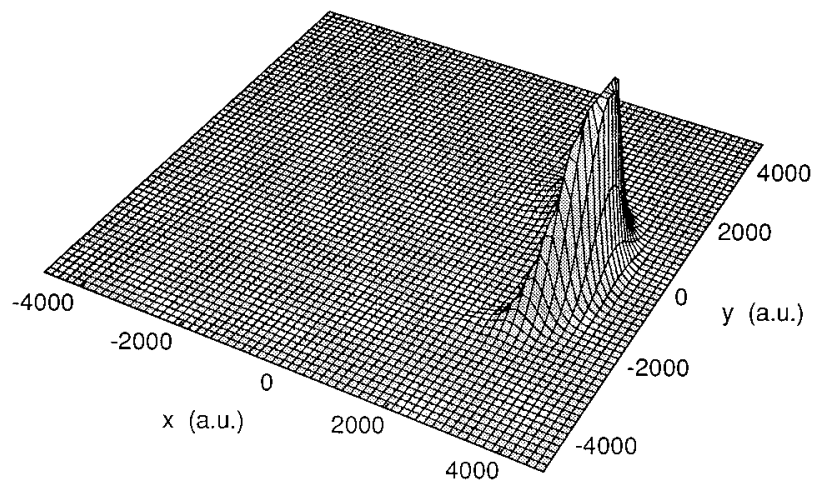

FIG. 2. Slices through the KSS wave packet in the $x-y$ plane, at different times during the classical orbital cycle. The quantity $r^{2}|\Psi(r, \theta, \phi, t)|^{2}$ (in arbitrary units) is shown as a function of $x$ and $y$ at the times (a) $t=0$, (b) $t=\frac{1}{3} T_{\mathrm{cl}}$, (c) $t=\frac{1}{2} T_{\mathrm{cl}}$, (d) $t=\frac{2}{3} T_{\mathrm{cl}}$, and (e) $t=T_{\mathrm{cl}}$.

modified. We choose $\left\langle p_{r}\right\rangle=0$ and $\left\langle L_{3}\right\rangle=\beta$, and specify $\Delta L_{3}$ as before. Denote by $E_{\bar{n} *}$ the energy expectation of the wave packet in the excited alkali-metal atom, and let $r_{\text {out }}^{*}$ be the outer apsidal point for a superposition of states with quantum-defect eigenenergies. Then, we impose $\langle H\rangle=E_{\bar{n}} *$ and $\langle r\rangle=r_{\text {out }}^{*}$, which differ from the hydrogenic case. To calculate $\langle H\rangle$ explicitly, we can take advantage of the completeness of the SQDT eigenfunctions and expand the initial KSS as a superposition of SQDT eigenstates,

$$
\Psi(r, \theta, \phi, 0)=\sum_{n, l, m} \tilde{c}_{n l m} R_{n} * l^{*}(r) Y_{l m}(\theta, \phi),
$$

where the expansion coefficients, which depend on the KSS parameters, can be determined by inversion. The expectation value for the Hamiltonian is then specified by

$$
\langle H\rangle=\sum_{n, l, m}\left|\tilde{c}_{n l m}\right|^{2} E_{n *}=E_{\bar{n} *} .
$$

It is known that the long-term revival times for an alkalimetal wave packet depend on the quantum defects and that the effects of the quantum defects are different from the effects of a laser detuning [25]. In additional, the appearance of deviations from the hydrogenic potential arising from the presence of core electrons in an alkali-metal atom means that 


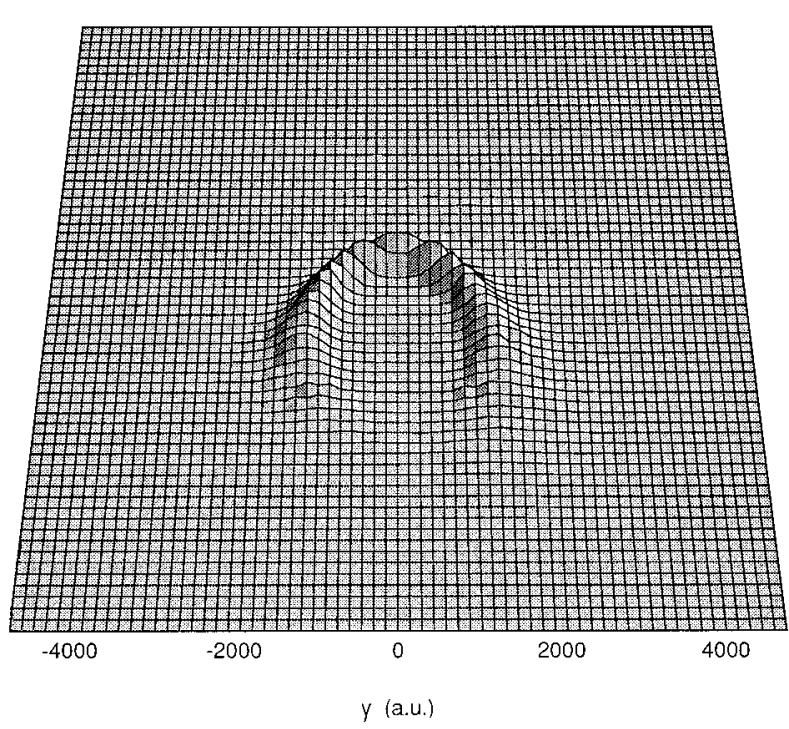

FIG. 3. The KSS wave packet at time $t=\frac{1}{2} T_{\mathrm{cl}}$, shown in Fig. 2(c), viewed from a point on the positive $x$ axis looking toward the nucleus.

the Runge-Lenz operator $\vec{A}$ does not commute with the Hamiltonian. The classical orbit therefore precesses at a rate determined by the quantum defect [12].

\section{SUMMARY}

In this paper, we have obtained minimum-uncertainty wave-packet solutions for the Schrödinger equation with a Coulomb potential in three dimensions. The solutions are the KSS and are given as a product of RSS and SSS. The RSS, previously derived, minimize the uncertainty relation for radial variables expressing the radial Coulomb problem in oscillator form. The SSS, constructed here, minimize the uncertainty relation for angular-coordinate and angularmomentum operators. The KSS provide analytical solutions to the Coulomb problem that move along classical Keplerian orbits. They exhibit both classical and quantum-mechanical features.

The KSS can be used as an analytical tool for studying the quantum-classical correspondence in the Coulomb problem. They may also be used to describe Rydberg wave packets created by excitation of a Rydberg atom with a short laser pulse in the presence of external fields. Such wave packets are expected to move in three dimensions along elliptical orbits that are strongly peaked around a plane. To match a KSS to a Rydberg wave packet, we choose the outer apsidal point as the initial location of the wave packet. The five KSS parameters are determined from the expectation values of the radial position, the radial momentum, the energy, the angular momentum $L_{3}$ transverse to the orbital plane, and the spread in $L_{3}$.

We obtained the time evolution of a KSS and provided an explicit example. The wave packet moves along an elliptical orbit with the classical Keplerian orbital period. The width of the wave packet oscillates during the motion, as is characteristic of a squeezed state. The KSS maintain their shape for several orbital cycles before collapsing and undergoing quantum recurrences.

Finally, we provided an extension of the construction to the case where quantum defects are present. With this analysis, the KSS can be used for the description of wave packets in alkali-metal atoms, which are the ones of choice in current experiments.

\section{ACKNOWLEDGMENT}

This work is supported in part by the National Science Foundation under Grant No. PHY-9503756.

\section{APPENDIX}

In this appendix, we discuss the functions $A_{j}^{\beta}(\delta)$ defined in Eq. (14) and examine some of their properties.

The integral in Eq. (14) can be evaluated. We find

$$
A_{j}^{\beta}(\delta)=4 \sqrt{\pi} \sum_{k=0}^{\beta} \frac{(-1)^{k}}{\delta^{k}}\left(\begin{array}{l}
\beta \\
k
\end{array}\right) \Gamma\left(k+\frac{1}{2}\right) i_{j+k}(2 \delta),
$$

where $i_{n}(z)$ is a modified spherical Bessel function. This exact expression permits the numerical computation of $A_{j}^{\beta}(\delta)$ to arbitrary precision using standard procedures.

A useful identity for the $A_{j}^{\beta}(\delta)$ can be derived by taking advantage of some properties of the modified spherical Bessel functions. We obtain

$$
A_{j}^{\beta+1}(\delta)=A_{j+2}^{\beta}(\delta)+\frac{j+1}{\delta} A_{j+1}^{\beta}(\delta) .
$$

For $j>0$, we find $A_{j}^{\beta}(0)=0$. For $j=0$, however, the value of the function with zero argument is

$$
A_{0}^{\beta}(0)=\frac{4 \pi(2 \beta) ! !}{(2 \beta+1) ! !} .
$$

These relations are used to simplify some expressions in the main body of the text.
[1] E. Schrödinger, Naturwissenschaften 14, 664 (1926).

[2] See, for example, Coherent States, edited by J. R. Klauder and B.-S. Skagerstam, (World Scientific, Singapore, 1985); Coherent States, edited by D. H. Feng, J. R. Klauder, and M. R. Strayer (World Scientific, Singapore, 1994).

[3] E. Schrödinger, Collected Papers on Wave Mechanics (Blackie, London, 1958).
[4] L. S. Brown, Am. J. Phys. 41, 525 (1973).

[5] J. Mostowski, Lett. Math. Phys. 2, 1 (1977).

[6] M. M. Nieto, Phys. Rev. D 22, 391 (1980); V. P. Gutschick and M. M. Nieto, ibid. 22, 403 (1980).

[7] D. S. McAnally and A. J. Bracken, J. Phys. A 23, 2027 (1990).

[8] J.-C. Gay, D. Delande, and A. Bommier, Phys. Rev. A 39, 6587 (1989). 
[9] M. Nauenberg, Phys. Rev. A 40, 1133 (1989).

[10] R. Bluhm, V. A. Kostelecký, and B. Tudose, Phys. Rev. A 52, 2234 (1995).

[11] R. Bluhm and V. A. Kostelecký, Phys. Rev. A 48, R4047 (1993).

[12] R. Bluhm and V. A. Kostelecký, Phys. Rev. A 49, 4628 (1994).

[13] V. A. Kostelecký and B. Tudose, Phys. Rev. A (to be published).

[14] A. Ten Wolde, L. D. Noordam, A. Lagendijk, and H. B. van Linden van den Heuvell, Phys. Rev. Lett. 61, 2099 (1988).

[15] J. A. Yeazell, M. Mallalieu, J. Parker, and C. R. Stroud, Phys. Rev. A 40, 5040 (1989).

[16] J. A. Yeazell, M. Mallalieu, and C. R. Stroud, Phys. Rev. Lett. 64, 2007 (1990).

[17] J. A. Yeazell and C. R. Stroud, Phys. Rev. A 43, 5153 (1991).

[18] D. R. Meacher, P. E. Meyler, I. G. Hughes, and P. Ewart, J. Phys. B 24, L63 (1991).

[19] J. Wals, H. H. Fielding, J. F. Christian, L. C. Snoek, W. J. van der Zande, and H. B. van Linden van den Heuvell, Phys. Rev. Lett. 72, 3783 (1994).

[20] Squeezed states are discussed, for example, in Proceedings of the Third International Workshop on Squeezed States and Uncertainty Relations, edited by D. Han, Y. S. Kim, and W. W. Zachary (NASA, Washington, D.C., 1994).

[21] J. Parker and C. R. Stroud, Phys. Rev. Lett. 56, 716 (1986); Phys. Scr. T12, 70 (1986).

[22] G. Alber, H. Ritsch, and P. Zoller, Phys. Rev. A 34, 1058 (1986); G. Alber and P. Zoller, Phys. Rep. 199, 231 (1991).
[23] I. Sh. Averbukh and N. F. Perelman, Phys. Lett. 139A, 449 (1989).

[24] M. Nauenberg, J. Phys. B 23, L385 (1990).

[25] R. Bluhm and V. A. Kostelecký, Phys. Rev. A 50, R4445 (1994).

[26] R. Bluhm and V. A. Kostelecký, Phys. Lett. A 200, 308 (1995); Phys. Rev. A 51, 4767 (1995).

[27] Z. D. Gaeta, M. Noel, and C. R. Stroud, Phys. Rev. Lett. 73, 636 (1994).

[28] For example, see the early review by P. Carruthers and M. M. Nieto, Rev. Mod. Phys. 40, 411 (1968).

[29] W. H. Louisell, Phys. Lett. 7, 60 (1963).

[30] V. A. Kostelecký and M. M. Nieto, Phys. Rev. Lett. 53, 2285 (1984); Phys. Rev. A 32, 1293, 3243 (1985). For a review, see V. A. Kostelecký, in Symmetries in Science VII: Dynamic Symmetries and Spectrum-Generating Algebras in Physics, edited by B. Gruber and T. Osaka (Plenum, New York, 1993).

[31] Our construction of the KSS for the hydrogen atom could in principle also be imitated for other coordinates separating the Schrödinger equation with a Coulomb potential. Some of these systems, in particular parabolic coordinates, have one or more interesting supersymmetries and an associated supersymmetrybased quantum-defect theory. However, to date only spherical polar coordinates involve an SQDT with an analytical and complete set of orthornormal eigenfunctions. Constructing the analogs of the KSS for alkali-metal atoms in other coordinate systems therefore is problematic. For a discussion of supersymmetries and SQDT in alternative coordinates, see R. Bluhm and V. A. Kostelecký, Phys. Rev. A 47, 794 (1993). 